\title{
Level of Patency for a One Year Period of Infra-inguinal Arterial Bypass in Patients with Peripheral Artery Disease \\ Nyityasmono Tri Nugroho**, Raden Suhartono ${ }^{a}$
}

Introduction: Peripheral arterial disease (PAD) is the most common macroangiopathic complication in type II diabetes mellitus, arising from inadequate blood sugar control. In the presence of PAD, the risk of limb loss will also increase, and arterial bypass is one method to reduce the risk of amputation. In Indonesia, the level of patency for the arterial bypass has not yet been published. On bypass with venous grafts, the patency rates at the location of infrapopliteal reach at $70-80 \%$, while the prosthetic graft is $30-50 \%$.

Method: From 2009 to 2012, patients with arterial bypasses were analyzed. The level of patency was described by ultrasound examination and pulsation on clinical examination in the distal anastomosis, reduced pain, and other examinations that support adequate revascularization. Identification of risk factors that affect patency, particularly protective risk factors, were also taken into account.

Results: From 2009 to 2012, 29 patients with infra-inguinal arterial bypass were collected. The ratio of men to women was $5: 1$, with a one-year patency rate of $88 \%$ in men, and $75 \%$ in women, for an overall of $86.2 \%$. The irreversible risk factor affecting patency was male ( $p$ $=0.117)$. Modifiable risk factors that decreasing patency level were smoking $(p=0.042)$ and more advanced stage of PAD ( $p=0.067)$. Smoking cessation $(p=0.041)$ and the use of drugs after bypass procedure $(p=0.072)$ were known to increase the level of patency.

Conclusion: The one-year patency rate for infra-inguinal artery bypass was $37-89 \%$. Smoking cessation was known to increase the level of patency.

Keywords: patency level, arterial bypass, infra-inguinal, peripheral arterial disease, diabetes https://doi.org/10.36864/jinasvs.2020.1.007
Copyright (C) 2020, The Indonesian Society for Vascular and Endovascular Surgery

JINASVS 2020;1(1):26-29

${ }^{*}$ Correspondence: yasmonn@gmail.com

aM.D., Vascular and Endovascular Division, Department of Surgery, Cipto Mangunkusumo Hospital - Faculty of Medicine, Universitas Indonesia, Jakarta, Indonesia

\section{INTRODUCTION}

Peripheral Arterial Disease (PAD) is a blockage of arterial blood flow out of coronary and intracranial arteries. PAD results from the process of atherosclerosis, embolism, thrombus, and inflammation that leads to arterial stenosis. ${ }^{1}$ As many as $3-10 \%$ of the world's population suffer asymptomatic PAD and rises to $15-20 \%$ in the population above 70 years. ${ }^{1,2}$ The presence of revascularization procedures in critical limb ischemia (CLI) significantly reduce amputation rates in PAD. Incidents for major amputation in large populations in several countries ranged from 120 to 500 million per year. The ratio between amputations below the knee and above the knee was about one to one. Approximately $60 \%$ of lower amputations were restored primarily, $15 \%$ restored secondarily, and another $15 \%$ had to be undergo amputations above the knee, and $10 \%$ were dead in the perioperative period. Our division in the vascular and endovascular, Department of Surgery FMUI - RSUPN Cipto Mangunkusumo, noted that $18.1-24.7 \%$ of diabetic patients with PAD had undergone amputation in the last three years, both for major and minor amputations. ${ }^{1,2}$

The Infra-inguinal bypass is a standard procedure for vascular surgery that has been developed in several decades. ${ }^{3}$ In the treatment of symptomatic PAD, the infra-inguinal arterial bypass is an effective procedure. ${ }^{4}$ This study aimed to know level of patency of one year in infra-inguinal arterial bypass and the factors that influence it.

\section{METHOD}

This was a prospective case-control study for patients in the Vascular and Endovascular Division of the Department of Surgery, Faculty of Medicine, Cipto Mangunkusumo, from January 2009 to December 2012. Data contained patient characteristics, risk factors, comorbid factors, and level of patency after infra-inguinal artery bypass were collected.

The data were colleceted from medical records from all of PAD patients in the Vascular and Endovascular Division of the Department of Surgery FKUI-RSUPN Cipto Mangunkusumo Jakarta. All PAD 
patients treated to the Vascular and Endovascular Division of the Department of Surgery, FKUI-RSUPN Cipto Mangunkusumo Jakarta, who underwent surgery for primary and secondary infra-inguinal artery bypass from of January 2009 to December 2012 were included. Exclusion criteria were patients who underwent endovascular revascularization before infra-inguinal arterial bypass surgery. The data were analyzed using SPSS statistical program version 11.5 for Windows $\AA$.

\section{RESULTS}

A total of 29 patients were included in this study; none of the study subjects dropped out. On the characteristics of respondents, variables such as age, sex, comorbid disease, risk factors, and surgical characteristics were identified (table 1).

On this table were presented the mortality and morbidity levels and the level of patency infrainguinal artery bypass (table 2 ).

In this study, the cumulative patency of bypass was examined using the Kaplan-Meier method and a comparison between survival graft using the Wilcoxon sign-rank test $(p<0.05$, figure 1 ). At the level of patency infra-inguinal artery bypass were obtained overall survival of $86.2 \%$, male overall survival of $88.0 \%$, and female overall survival of $75 \%(p=0.8615$,log rank test)

\section{DISCUSSION}

The prevalence of peripheral arterial disease (PAD) ranges between $3-10 \%$ and increases as the life expectancy rate. At the age of 70 years and above, the prevalence of PAD also increases to 15$20 \%{ }^{1,2,5,6}$ In this study, the increasing trend in the prevalence paralleled with the age growth. At the age of 40-49 years, the prevalence was $6.8 \%$, after the following decade increased by $27.5 \%$, and the following decade increased again by $44.8 \%$, even though at the age of $>70$ years it was only $3.4 \%$. In terms of sex ratio, more men suffer from PAD than women (up to $5: 1$ ). Comorbid factors for diabetes mellitus and risk factors for smoking become the first factors for influencing PAD. $1,2,5,6$
Revascularization in peripheral arterial disease (PAD) can be done endovascularly or bypass surgery. Bypass procedure performed at the infrainguinal level can be done in three places, which are femoropopliteal, femorodistal, and poplitealdistal. At the level of femoropopliteal and femorodistal, the patency was $77 \%$ and $67 \%$, respectively. ${ }^{7}$ In this study, the patency was $86.2 \%$, which consists of $88 \%$ in men, and $75 \%$ in women. This result was consistent with the literature, which said that both PTFE graft and great saphenous vein graft had patency ranging from $75-84 \%$ for the patency rate of 1-year primary patency. For secondary patency, infra-inguinal femoropopliteal was not stated, but in the below-knee femoropopliteal there was a $68-96 \%$ patency rate. ${ }^{4}$

Factors affecting the output of infra-inguinal bypass are the presence of coronary heart disease, diabetes, renal insufficiency, age, smoking, chronic obstructive pulmonary disease, hypercoagulability, and the degree of ischemia. ${ }^{8,9}$ In this study, smoking and diabetes became the first risk factors and comorbid affecting the output of the bypass.

In this study, we only got 29 samples, which was considered as a small amount in researching a survival analysis case. However, the results obtained were similar to the large-scale research.

The revascularization process in PAD does not only lead to survival analysis, but also the benefits felt by the patient. It was found that there was a decrease in the intensity of claudication and improvement in the condition of the wound or ulcer, so it can be concluded that revascularization also provides a clinical improvement for patients (table 2).

Several studies stated that there are relationship between risk factors and high amputation rates, including the Finnvasc score, which consists of diabetes, coronary heart disease, leg gangrene, and immediate surgery. This score has an effect on morbidity, which is the rate of amputations. Thus, it can determine the need for revascularization. Other studies mention Prevent III (PIII) risk factors, which are dialysis (4 points), tissue loss ( 3 points), age $>75$ years ( 2 points),

Table 1. Table of characteristics of PAD infra-inguinal artery bypass patients.

\begin{tabular}{llll}
\hline & Classification & Number $(\%)$ & $\boldsymbol{p}$ value \\
\hline Age & $\leq 30$ & $1(3,4)$ & \\
& $30-39$ & $4(13,7)$ & \\
& $40-49$ & $2(6,8)$ & 0,062 \\
\hline Sex & $50-59$ & $8(27,5)$ & \\
& $60-69$ & $13(44,8)$ & 0,117 \\
\hline Comorbid & Men & $1(3,4)$ & 0,056 \\
diseases & Women & $25(86,2)$ & 0,069 \\
& Diabetes mellitus & $27(93,1)$ & 0,198 \\
& Kidney & $3(10,3)$ & 0,083 \\
& Heart & $6(20,6)$ & - \\
\hline Risk factors & Lung & $4(13,7)$ & 0,042 \\
& No comorbid & $0(0)$ & 0,096 \\
& Smoking & $15(51,7)$ & 0,106 \\
& Diabetes mellitus & $27(93,1)$ & 0,112 \\
\hline Anastomotic & Hypercholesterolemia & $19(65,5)$ & \\
site & Hypertension & $23(79,3)$ & 0,077 \\
\hline Conduit type & Femoro-popliteal & $14(48,2)$ & \\
& Femoro-distal & $12(41,3)$ & 0,098 \\
\hline
\end{tabular}


Table 2. Mortality and morbidity rates and patency rates for arterial bypass.

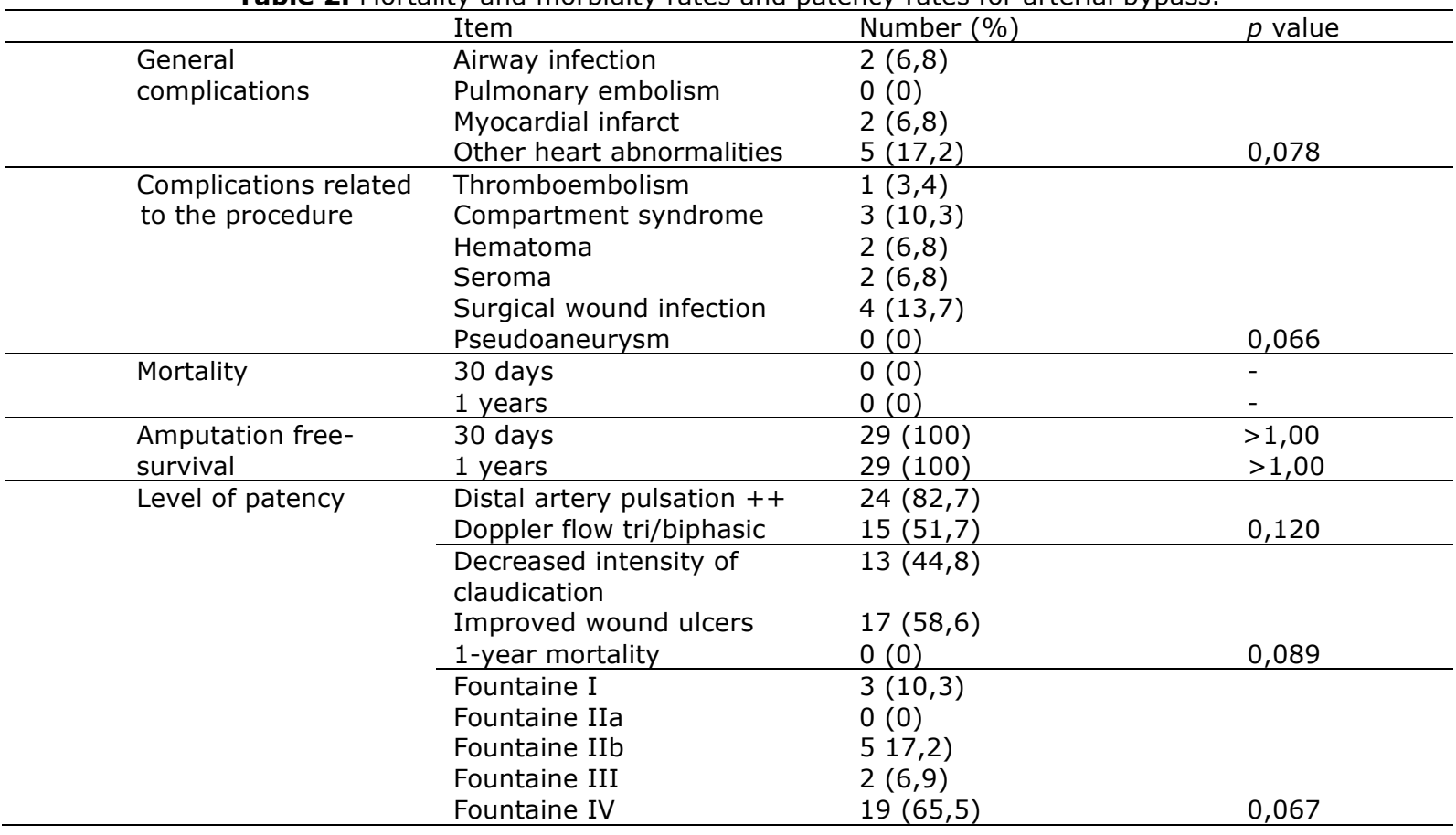

Survival Function

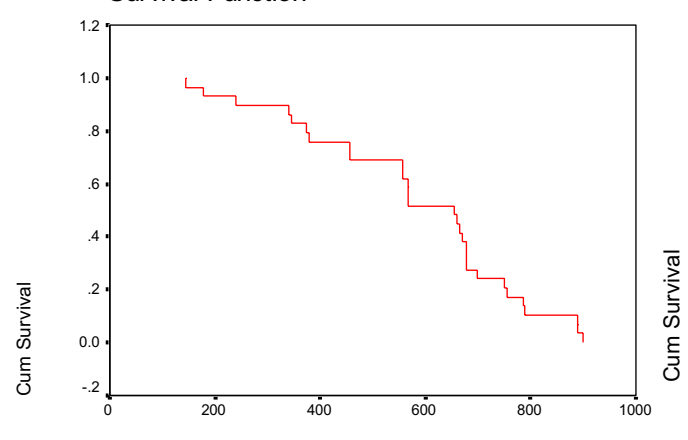

PATENCY
Survival Functions

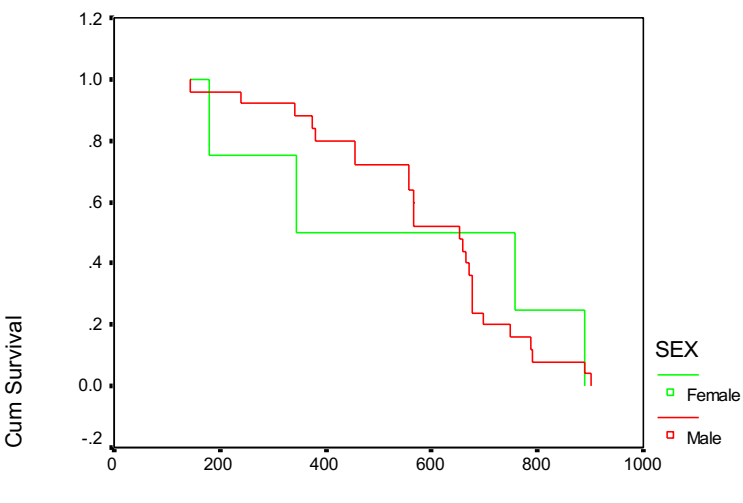

PATENCY

Figure 1. Overall survival chart of patency levels for infra-inguinal artery bypass and each sex.

hematocrit $<30$ ( 2 points), and coronary heart disease ( 1 point).

Revascularization is also affected by the type of conduit used. All studies support autologous reverse vein as the standard for revascularization, but in some studies, it has a similar survival rate with venous and synthetic grafts.

\section{CONCLUSION}

As people age more than 40 years are increasing, the prevalence of PAD will also increase, with men are more frequent compared to women ( 5 : 1 ratio). The dominant risk factors and comorbid factors were diabetes and smoking. The patency rate for infra-inguinal artery bypass was $86.2 \%$ for overall survival, $88 \%$ for men, and $75 \%$ for women. There was a significant relationship with smoking cessation with the level of patency.

\section{ACKNOWLEDGMENTS}

The author states the original work, and there is no conflict of interest in doing this research.

\section{ORCID ID OF AUTHORS}

Nyityasmono Tri Nugroho

https://orcid.org/0000-0001-8876-9698

Raden Suhartono

https://orcid.org/0000-0002-9142-3062

\section{REFERENCES}

1. Lars Norgen, William R Hiatt (editor). Inter society consensus for the management of peripheral arterial disease (TASC II). TASC II Working Group. 2004. 
2. Vivian Ho, Douglas Wirthlin, Huifeng Yun, Jeroan Allison. Physician suplly, treatment, and amputation rates for peripheral arterial disease. J Vasc Surgery 2005; 42:81-7

3. T Lees, T Troeng, I. A Thomson, et al. International variations in infrainguinal bypass surgery - A Vascunet Report. Eur J Vasc Endovasc Surg. 2012 Aug;44(2):185-92. doi: 10.1016/j.ejvs.2012.05.006.

4. Eva Arvela. Editor. High-risk patients and high-risk grafts in infrainguinal bypass for critical limb ischemia. Academic Dissertation. Department of Surgery, Vascular Surgery, Institut of Clinical Medicine. University of Helsinki. 2011.

5. Paul L Allan. The peripheral arteries. In: Paul L Allan, Paul A Dubblins, Myron A Pozniak. W Norman McDicken. Clinical Doppler Ultrasound. Ch 4. Churchill Lkivingstone-Elsevier. London: 2004. p. 65-86

6. R Eugen Zierlier. Ultrasound assessment of lower extremity arteries. In: Zwiebel, Pellerto. Introduction to vascular ultrasonography $5^{\text {th }}$ ed. Ch 18 . Elsevier-Saunders. Philadelphia:2005, p. 341-380

7. Pereira et al. Meta-analysis of femoropopliteal bypass grafts for lower extremity arterial insufficiency. J Vasc Surg 2006;44:510-7

8. Sarin S, Shami S, Shields DA, Scurr JH, Smith PD. Selection of amputation level: a review. Eur J Vasc Surg. 1991. Dec;5(6):611-20

9. Patrick D Hallihan, Niamh Ni Choileain, eddie Myers, et al. Predictors Predictors of time to graft failure following infrainguinal arterial reconstruction. Surgical Science, 2011, 2, 166-172. 